\title{
Accuracy of Freehand versus Navigated Thoracolumbar Pedicle Screw Placement in Patients with Metastatic Tumors of the Spine
}

\author{
Rafael De La Garza Ramos, ${ }^{1,2}$ Murray Echt,, Joshua A. Benton, ${ }^{1}$ Yaroslav Gelfand, ${ }^{1,2}$ Michael Longo, ${ }^{1}$ Vijay Yanamadala, ${ }^{1,2}$ \\ Reza Yassari ${ }^{1,2}$ \\ Spine Research Group,' Montefiore Medical Center/Albert Einstein College of Medicine, Bronx, NY, USA \\ Department of Neurological Surgery, ${ }^{2}$ Montefiore Medical Center/Albert Einstein College of Medicine, Bronx, NY, USA
}

Objective : To compare the accuracy and breach rates of freehand (FH) versus navigated (NV) pedicle screws in the thoracic and lumbar spine in patients with metastatic spinal tumors.

Methods : A retrospective review of adult patients who underwent pedicle screw fixation in the thoracic or lumbar spine for metastatic spinal tumors between 2012 and 2018 was conducted. Breaches were assessed based on the Gertzbein and Robbins classification and only screws placed $>4 \mathrm{~mm}$ outside of the pedicle wall (lateral or medial) were considered breached.

Results : A total of 62 patients received 547 pedicle screws (average 8 per patient) - 34 patients received 298 pedicle screws in the FH group and 28 patients received 249 screws in the NV group. There were 40/547 breaches, corresponding to a breach and accuracy rate of $7.3 \%$ and $92.7 \%$, respectively. The breach rate was $9.7 \%$ in the $\mathrm{FH}$ group and $4.4 \%$ in the NV group (chi-squared test, $p=0.017$ ); this corresponded to an accuracy rate of $90.3 \%$ and $95.6 \%$, respectively. Only one patient from the overall cohort (in the FH group) required revision surgery due to a medial breach abutting the spinal cord ( $1.6 \%$ of all patients; $2.9 \%$ of $\mathrm{FH}$ patients); no patient suffered organ, vessel, or neurological injury from screw breaches.

Conclusion : Navigated pedicle screw placement in patients with metastatic spinal tumors has a significantly higher radiographic accuracy compared to the FH technique. However, the revision surgery was low and no patient suffered from clinically-relevant breach. Navigation also offers the advantage of real-time localization of spinal tumors and aids in targeting and resection of these lesions.

Key Words : Spinal fusion · Instrumentation · Pedicle screws · Spinal neoplasms.

\section{INTRODUCTION}

The use of pedicle screw fixation is considered the "gold standard" in posterior instrumentation techniques in the tho- racolumbar spine $e^{4)}$. Patients with metastatic tumors to the vertebral column may require fixation after tumor resection or due to intrinsic tumor-related spinal instability. Although still considered mostly palliative in nature except in select cas-

- Received : December 31, $2019 \bullet$ Revised : March 19, 2020 •Accepted : March 25, 2020

- Address for reprints : Rafael De La Garza Ramos

Spine Research Group, Montefiore Medical Center/Albert Einstein College of Medicine, 3rd floor, 3316 Rochambeau Avenue, Bronx, NY 10467, USA

Tel : +1-718-920-4216, Fax : +1-718-547-4591, E-mail : rafdelag@gmail.com, ORCID : https://orcid.org/0000-0002-5536-2514

This is an Open Access article distributed under the terms of the Creative Commons Attribution Non-Commercial License (http://creativecommons.org/licenses/by-nc/4.0) which permits unrestricted non-commercial use, distribution, and reproduction in any medium, provided the original work is properly cited. 
es, surgery and multidisciplinary care for metastatic disease to the spine has allowed for patients to live longer with acceptable qualities of life. In these cases, achieving adequate fixation and possible arthrodesis is important for good long-term results ${ }^{5)}$.

Pedicle screw insertion can be done using traditional anatomical landmarks via the freehand $(\mathrm{FH})$ technique or with use of intraoperative navigation (NV), among others. Multiple studies have shown improved radiographic accuracy with navigated screws, but the majority of these studies have combined results and included patients with degenerative spine disease, trauma, idiopathic deformity, and to a lesser degree on tumor cases ${ }^{7,9,17,22)}$. Although the pedicle screw insertion technique does not vary based on the underlying pathology, the major challenge of placing pedicle screws in patients with metastatic disease is bone quality and potential higher risk for pedicle fractures. Thus, the purpose of this study was to compare the FH technique to a three-dimensional NV technique and assess pedicle screw breaches in patients with secondary malignant tumors of the thoracic and lumbar spine.

\section{MATERIALS AND METHODS}

\section{Study design and data source}

This study received approval by the Institutional Review Board of Montefiore Medical Center (2016-6896). We reviewed our neurosurgical spine operative database for the years 2012 through 2018 to identify all patients who underwent surgery for metastatic spinal tumors. Inclusion criteria were 1) patients who underwent pedicle screw placement in the thoracic or lumbar spine via a FH or NV technique; and 2) patients with complete postoperative computed tomography (CT) scans to allow assessment of breaches. Reviewed and examined patient data included age, sex, number of pedicle screws placed, spinal level of placement, number of laminectomy levels, use of transpedicular corpectomy, use of cement augmentation, estimated blood loss, and operative time.

$\mathrm{FH}$ pedicle screw placement in the thoracic spine was done using anatomical landmarks and the "superior facet rule", with an entry point 2-3 mm lateral to the midline of the superior articulating facet and within the superior $1 / 3$ of the transverse process $^{16)}$. Angulation in the sagittal and axial plane was done in a conventional fashion based on pedicle morpholo- $\mathrm{gy}^{12)}$. For pedicle screws in the lumbar spine, these were placed at the intersection of the pars, the transverse process, and the mamillary process of each vertebral body. For the NV technique, we used the $\mathrm{O}-\mathrm{arm}^{\circledR}$ system (Medtronic, Minneapolis, MN, USA) to acquire an intraoperative CT scan, which was then transferred to the StealthStation ${ }^{\mathrm{TM}}$ Surgical Navigation System (Medtronic, Minneapolis, MN, USA). A navigated probe was used to select the starting point, followed by use of a navigated pedicle finder to cannulate the pedicle. Tunnels were checked with a ball-tipped feeler and screws were placed also in a navigated fashion. The decision to employ the $\mathrm{FH}$ versus the NV technique was surgeon-dependent, but for the most part the most recent cases (after 2014) were NV cases.

\section{Outcome variables}

Our main outcome variable was screw breach. This was assessed via the Gertzbein and Robbin's classification, which assigns breach grades based on the number of millimeters the screw is outside of the pedicle wall ${ }^{8)}$. Although breaches are assigned grades by $2 \mathrm{~mm}$ increments, screws that are $>4 \mathrm{~mm}$ beyond the pedicle wall are considered potentially "unsafe" given proximity to neural elements or other organ/vascular structures ${ }^{8,13)}$. Therefore, we only categorized screws placed $>4$ $\mathrm{mm}$ beyond the pedicle wall as breached. Conversely, an "accurate" screw as considered as such if no breach was detected at all or when the breach was $<4 \mathrm{~mm}$ outside of the pedicle.

Secondary outcome measures included need for revision surgery and/or other complications due to screw misplacement.

\section{Statistical analysis}

All analyses were performed in Stata SE 12 (StataCorp, College Station, TX, USA). Two groups were established for analysis : the FH and NV technique groups. Descriptive statistics were performed to describe the study population as well as total number of screws, breaches, and accuracy. Means were compared between groups via t-tests and frequencies via chisquared tests. Statistical significance was defined as a probability value $<0.05$.

\section{RESULTS}

A total of 62 patients with metastatic tumors of the spine 
and thoracolumbar instrumentation at our institution between 2012 and 2018 were identified and met inclusion criteria for our study. The primary tumor locations in descending order of frequency were : breast (17.7\%), hematopoietic (17.7\%), prostate $(16.5 \%)$, other $(14.5 \%)$, kidney $(9.7 \%)$, colorectal (8.1\%), liver (8.1\%), lung (4.8\%), and gynecological in $3.2 \%$ of cases.

The average age of all patients was 58.7 years (standard deviation [SD], 1.8) and 58.1\% were male (Table 1) - patients in the NV group were significantly older compared to the $\mathrm{FH}$ group ( $p=0.003$ ). There were no significant differences in gen$\operatorname{der}(p=0.053)$, average number of screws $(p=0.863)$, number of laminectomy levels ( $p=0.618$ ), percentage of patients who underwent transpedicular corpectomy $(p=0.167)$, use of cement augmentation ( $p=0.293)$, average blood loss $(p=0.792)$, or average operative time $(p=0.453)$.

A total of 547 screws were analyzed (average 8.8 screws per patient) -298 in the FH group and 249 in the NV cohort. The five most common spinal levels of placement were T6 (63 screws; 11.5\%), T7 (55 screws; 10.1\%), T9 (51 screws; 9.3\%), T5 (49 screws, 9.0\%), and T8 (48 screws; 8.8\%) (Fig. 1). The proportion of screws placed on each level was not significantly different between the FH and NV groups (all $p>0.05$ ).

There were a total of 40/547 breaches (7.3\%), which corre-

Table 1. General and operative characteristics of 62 patients who underwent pedicle screw fixation for metastatic tumors of the spine in the freehand $(\mathrm{FH})$ and navigated (NV) groups

\begin{tabular}{lcccc}
\hline Variable & All patients & FH group & NV group & p-value \\
\hline Number of patients & 62 & 34 & 28 & 64.6 \\
Mean age (years) & 58.7 & 53.9 & 71.4 & 0.003 \\
Male sex (\%) & 58.1 & 47.1 & 8.9 & 0.053 \\
Average number of screws & 8.8 & 8.8 & 1.9 & 0.863 \\
Average number of laminectomy levels & 2.0 & 2.0 & 52.6 & 0.618 \\
Transpedicular corpectomy (\%) & 62.9 & 70.6 & 10.7 & 0.167 \\
Cement augmentation (\%) & 16.1 & 20.6 & 719 & 0.293 \\
Average EBL (mL) & 742 & 762 & 7.4 & 0.792 \\
Average operative time (hours) & 7.2 & 6.9 & 0.435 \\
\hline
\end{tabular}

EBL : estimated blood loss

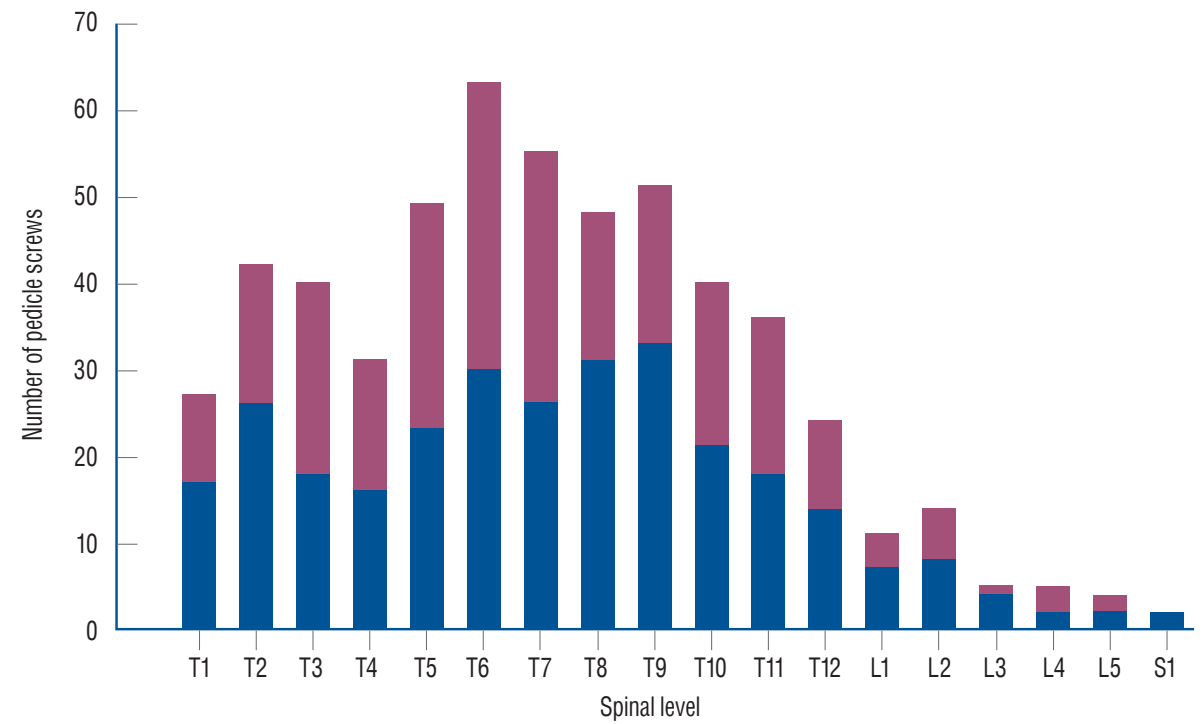

Fig. 1. Distribution of 547 pedicle screws placed in 62 patients with metastatic tumors of the spine. The distribution of screw placement by the freehand (blue) and navigated (red) technique was not significantly different (all $p>0.05$ ). 
sponded to an overall accuracy of $92.7 \%$. The five most common involved levels were the following : $\mathrm{T} 7$ (10 breaches, $25.0 \%$ of all breaches), T9 (seven breaches, 17.5\%), T5 (five breaches, $12.5 \%$ ), T4 (four breaches, 10.0\%), and T3, T6, and T8 (each with three breaches, 7.5\% of all breaches each) (Fig. 2).

The breach rate was 29/298 (9.7\%) in the FH group and $11 / 249$ (4.4\%) in the NV group (chi-squared test, $p=0.017$ ); this corresponded to an accuracy rate of $90.3 \%$ and $95.6 \%$, respectively. Out of the 40 breaches, 34/40 (85\%) were lateral and 6/40 (15\%) were medial (Fig. 3, left). There was one occurrence of a "superolateral breach", which was analyzed in the "lateral breach group; there were no inferior breaches. Only one patient (1.8\% of all patients; $0.2 \%$ of all screws) required revision of one medial breach. This patient had a medial breach at T6 abutting the spinal cord (Fig. 3, right) but without causing any neurological injury; the decision was made to revise the screw the morning after the index procedure. No patient in our series experienced organ, vascular, or neurological injury from any misplaced screw.

\section{Screw breach details}

Although we only considered screw breaches $>4 \mathrm{~mm}$ be-

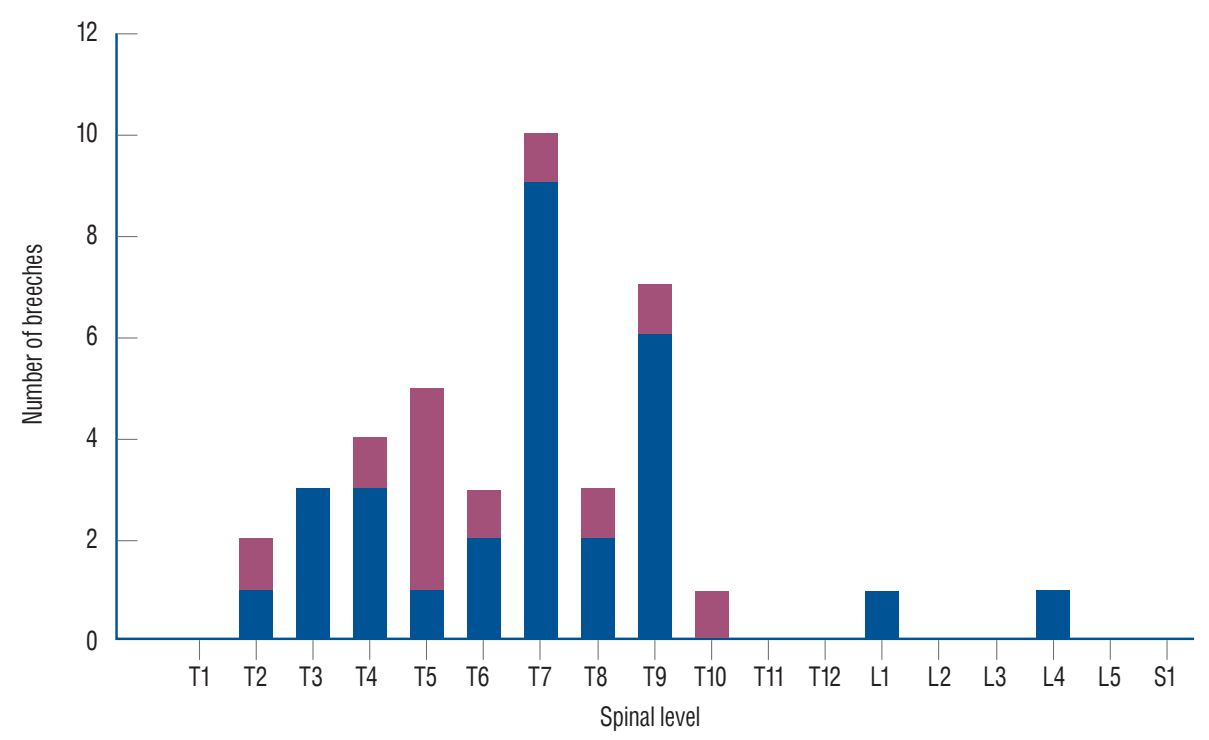

Fig. 2. Distribution of 47 pedicle screws breaches in 62 patients with metastatic tumors of the spine. The distribution of screw placement by the freehand (blue) and navigated (red) technique was not significantly different (all $p>0.05$ ).
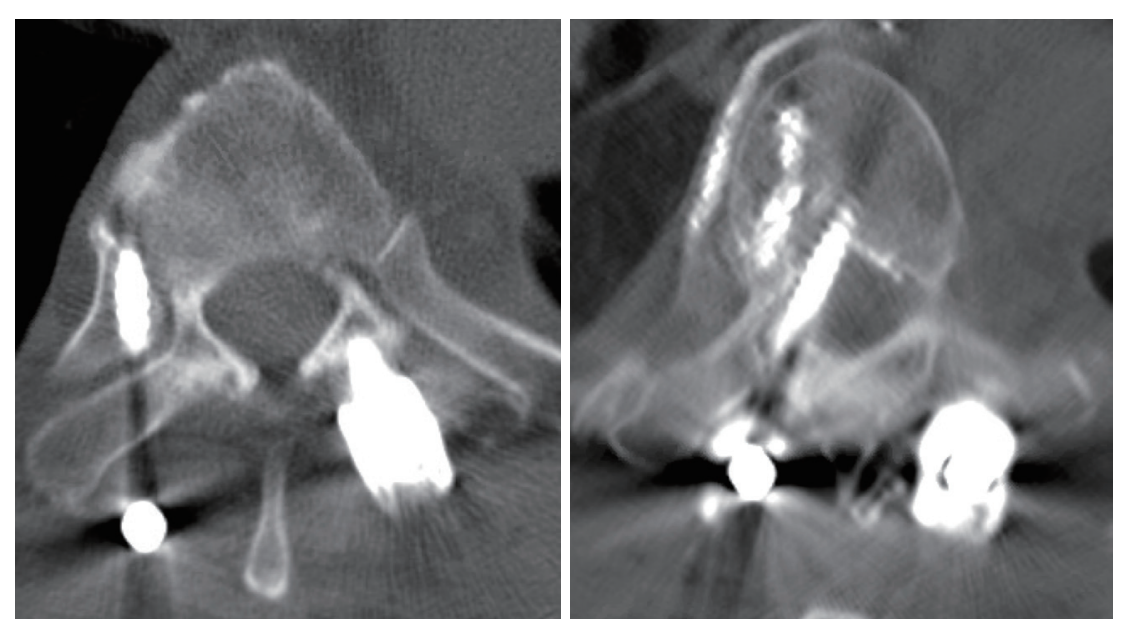

Fig. 3. Postoperative computed tomography scans showing examples of a lateral breach (left) and a medial breach (right). The patient with the medial breach was the only patient in our study who underwent revision due to close proximity to the spinal cord; no neurological sequelae occurred. This patient also had some extravasation of cement into the adjacent vasculature without any sequelae. 
yond the pedicle for our primary outcome measure, there were 23 breaches less than $2 \mathrm{~mm}$ beyond the pedicle and 39 breaches $2-4 \mathrm{~mm}$ beyond the pedicle. Stratified by operative technique, there were 16 and seven breaches less than $2 \mathrm{~mm}$ in the FH and NV groups, respectively. Conversely, there were 22 and 17 breaches $2-4 \mathrm{~mm}$ beyond the pedicle in the $\mathrm{FH}$ and NV groups.

\section{Other instrument-unrelated surgical complica- tions}

A total of 26 patients experienced at least one perioperative complication (41.9\%). These included deep vein thrombosis (12.9\%), pneumonia (8.1\%), wound infection $(4.8 \%)$, pulmonary embolism (4.8\%), urinary tract infection $(3.2 \%)$, urinary retention $(3.2 \%)$, bowel obstruction (1.6\%), and new neurological deficit (1.6\%).

\section{DISCUSSION}

The use of pedicle screws for internal fixation in metastatic disease allows for restoration of spinal instability, pain relief, and correction of deformity ${ }^{5}$. The use of the FH technique for screw insertion requires thorough knowledge of anatomical landmarks and pedicle morphology, with accuracy rates reported to be above $90 \%$ in most investigations ${ }^{11,12,14,20)}$. The use of intraoperative 3-D navigation, on the other hand, has been shown to be superior in terms of radiographic accuracy ${ }^{79,17,22)}$. In spinal oncology, this technique also offers the advantage of real-time localization and also aids in tumor resection. In this study, we conducted a retrospective review to determine the accuracy rate of $\mathrm{FH}$ and $\mathrm{NV}$ pedicle screw placement in patients with spinal tumors, finding that NV screws had a significantly higher radiographic accuracy rate $(95.6 \%$ vs. $90.3 \%)$. Fortunately, none of the breached screws in any group resulted in nerve, vascular, or organ injury.

As mentioned above, radiographic accuracy of screws placed with the NV technique has been shown to be superior to the FH technique previously. Shin et al. ${ }^{22)}$ conducted a systematic review and meta-analysis on the matter, which included a review of 18 cohort studies and two randomized trials published between the years 2000 and 2011. A total of 8539 screws placed for degenerative disease, trauma, infection, and tumors were analyzed (3725 in the FH group and 4814 in the
NV group), finding a 15\% risk of perforation in the FH group and $6 \%$ in the NV cohort ${ }^{22}$. No neurological complications were found in the NV group, whereas three neurological complications were reported in the FH cohort. However, no differences in revision surgery rates were found.

In patients with spinal tumors, anatomy may often be disrupted due to loss of bone integrity, deformity, or direct tumor invasion. Although spinal segments directly affected by tumors are rarely instrumented, the adjacent levels oftentimes suffer from poor bone quality or deformity. While there is vast literature on insertion of pedicle screws for degenerative disease, trauma, and non-tumor related deformity, few studies have focused solely on pedicle screw fixation in the setting of malignant tumors ${ }^{5}$. Fourney et al. ${ }^{5}$ described 100 cases of pedicle screw instrumentation in metastatic disease performed at MD Anderson Cancer Center between 1994 and 1999. They were amongst the first to report a large series of patients, placing a total of 514 pedicle screws (median four per case, most common level L2). Surgery was found to provide significant pain relief, significant improvement in neurological function, and without any complication directly related to screw placement ${ }^{5}$.

Accurate placement of pedicle screws is important biomechanically and in order to avoid potential injury to critical neurovascular structures and organs such as the lung or viscera. An optimal screw is placed parallel to the endplate, fills approximately $80 \%$ of the pedicle diameter, and is inserted at a depth of $80 \%$ into the vertebral body for maximal pullout strength $^{3,15)}$. More importantly, breached screws or "airball" screws (screws that miss the vertebral body) are known to have considerably decreased pullout strengths compared to screws placed entirely within the pedicle ${ }^{2)}$. Although rare, misplacement of screws can lead to nerve root irritation, spinal cord injury, cerebrospinal fluid leak, pneumothorax, vascular injury (aorta or vena cava), and others ${ }^{6}$.

Ultimately, however, misplaced/breached screws are extremely unlikely to cause clinically relevant injuries. In a literature review of 35630 pedicle screws, Gautschi et al. ${ }^{6}$ found a nerve root irritation rate of $0.19 \%$ and $<0.01 \%$ risk of injury to the pleura. Injury to the aorta is even less common, with only a handful of cases reported in the literature ${ }^{10)}$. Although screws can potentially be in close proximity or even in tight contact with a major vessel, removal of asymptomatic screws is controversial and should be done on a case-by-case basis ${ }^{6}$.

Radiographic accuracy was shown to be superior for pa- 
tients who received NV screws versus FH screws in our study. Although only one screw revision was made and no cases of neurovascular/organ injury occurred in either group, we believe that the use of navigation has other important advantages over the FH technique in spinal tumor surgery. Nasser et al. ${ }^{19)}$ demonstrated the use of image-guided navigation for biopsy/resection of spinal column tumors in 50 patients. Navigation tools not only allow for placement of pedicle screws, but also allow for real-time localization, tracking, excision, and determination of resection margins ${ }^{1,19,21)}$. Furthermore, with the advent of minimally invasive techniques, the use of 3-D navigation allows for placement of navigated screws with or without cement augmentation by using small incisions and minimal dissection of soft tissue and muscle ${ }^{18)}$.

The breach rates reported in our study are consistent with rates reported in degenerative spine disease, trauma, and deformity, which suggests that the underlying pathology does not necessarily impact the accuracy rate of screw placement and is a reassuring finding. While the $\mathrm{FH}$ technique should be the first step in learning how to place pedicle screws in the spine, NV is an available tool that can also be considered when treating patients with spinal lesions. The main objective of this investigation was to compare techniques in the tumor population, which had not been done previously.

Although we demonstrated a significantly higher radiographic accuracy rate for NV screws compared to the $\mathrm{FH}$ technique in patients with spinal tumors, our study has several limitations. One of the most important issues when performing studies related to screw accuracy is the definition of breaches. While we utilized the widely-accepted Gertzbein and Robbins classification, a recent systematic review found more than 30 different classification systems ${ }^{13)}$. This could potentially make a generalizability of our findings difficult if different breach definitions are used in other studies. In addition, our series is a single-center experience and this creates a potential risk of bias. Future multi-center studies or metaanalyses may further corroborate our findings for patients with metastatic spinal disease.

\section{CONCLUSION}

Pedicle screw fixation in patients with metastatic tumors allows for treatment of instability, pain relief, and deformity correction. In this study, the use of intraoperative navigation was associated with a significantly higher radiographic accuracy compared to the FH technique. Although no breaches resulted in a clinically relevant complication (other than one screw revision), the use of the NV technique may be preferred, including the ability for real-time tumor localization and aid in resection.

\section{CONFLICTS OF INTEREST}

No potential conflict of interest relevant to this article was reported.

\section{INFORMED CONSENT}

Informed consent was obtained from all individual participants included in this study.

\section{AUTHOR CONTRIBUTIONS}

\author{
Conceptualization : RDLGR, RY \\ Data curation : RDLGR, ME \\ Formal analysis : RDLGR \\ Methodology : RDLGR, ME, JAB, YG \\ Project administration : VY, RY \\ Visualization : RDLGR \\ Writing - original draft : RDLGR, UE, JAB, ML, VY, RY \\ Writing - review \& editing: RDLGR, UE, JAB, ML, VY, \\ RY
}

\section{ORCID}

RafaelDeLaGarzaRamos https://orcid.org/0000-0002-5536-2514

Murray Echt https://orcid.org/0000-0002-4504-4918

Joshua A. Benton https://orcid.org/0000-0001-8854-7155

Yaroslav Gelfand https://orcid.org/0000-0002-0920-8911

Michael Longo https://orcid.org/0000-0002-2462-6118

Vijay Yanamadala https://orcid.org/0000-0002-2456-5888

Reza Yassari https://orcid.org/0000-0002-2593-2635 


\section{- Acknowledgements}

This work was presented as an oral presentation in the 2019 Global Spine Congress in Toronto, Canada, on May 15-18, 2019.

\section{References}

1. Arand M, Hartwig E, Kinzl L, Gebhard F : Spinal navigation in tumor surgery of the thoracic spine: first clinical results. Clin Orthop Relat Res (399) : 211-218, 2002

2. Brasiliense LB, Theodore N, Lazaro BC, Sayed ZA, Deniz FE, Sonntag VK, et al. : Quantitative analysis of misplaced pedicle screws in the thoracic spine: how much pullout strength is lost?: presented at the 2009 Joint Spine Section Meeting. J Neurosurg Spine 12 : 503-508, 2010

3. Cho W, Cho SK, Wu C : The biomechanics of pedicle screw-based instrumentation. J Bone Joint Surg Br 92 : 1061-1065, 2010

4. duPleiss SJ, Hurlbert RJ : Pedicle screw fixation of the thoracic and lumbar spine in Dickman CA, Fehlings MG, Gokaslan ZL (eds) : Spinal Cord and Spinal Column Tumors. New York : Thieme, 2006, pp587-598

5. Fourney DR, Abi-Said D, Lang FF, McCutcheon IE, Gokaslan ZL : Use of pedicle screw fixation in the management of malignant spinal disease: experience in 100 consecutive procedures. J Neurosurg 94(1 Suppl) : 25-37, 2001

6. Gautschi OP, Schatlo B, Schaller K, Tessitore E : Clinically relevant complications related to pedicle screw placement in thoracolumbar surgery and their management: a literature review of 35,630 pedicle screws. Neurosurg Focus 31 : E8, 2011

7. Gelalis ID, Paschos NK, Pakos EE, Politis AN, Arnaoutoglou CM, Karageorgos AC, et al. : Accuracy of pedicle screw placement: a systematic review of prospective in vivo studies comparing free hand, fluoroscopy guidance and navigation techniques. Eur Spine J 21 : 247-255, 2012

8. Gertzbein SD, Robbins SE : Accuracy of pedicular screw placement in vivo. Spine (Phila Pa 1976) 15 : 11-14, 1990

9. Innocenzi G, Bistazzoni S, D'Ercole M, Cardarelli G, Ricciardi F : Does navigation improve pedicle screw placement accuracy? Comparison between navigated and non-navigated percutaneous and open fixations. Acta Neurochir Suppl 124 : 289-295, 2017

10. Kakkos SK, Shepard AD : Delayed presentation of aortic injury by pedicle screws: report of two cases and review of the literature. J Vasc Surg 47 : 1074-1082, 2008

11. Karapinar L, Erel N, Ozturk H, Altay T, Kaya A : Pedicle screw placement with a free hand technique in thoracolumbar spine: is it safe? J Spinal Disord Tech 21 : 63-67, 2008

12. Kim YJ, Lenke LG, Bridwell KH, Cho YS, Riew KD : Free hand pedicle screw placement in the thoracic spine: is it safe? Spine (Phila Pa 1976) 29 : 333-342; discussion 342, 2004

13. Kosmopoulos V, Schizas C : Pedicle screw placement accuracy: a metaanalysis. Spine (Phila Pa 1976) 32 : E111-E120, 2007

14. Kotil K, Bilge $T$ : Accuracy of pedicle and mass screw placement in the spine without using fluoroscopy: a prospective clinical study. Spine J 8 : 591-596, 2008

15. Krag MH, Beynnon BD, Pope MH, Frymoyer JW, Haugh LD, Weaver DL : An internal fixator for posterior application to short segments of the thoracic, lumbar, or lumbosacral spine. Design and testing. Clin Orthop Relat Res (203) : 75-98, 1986

16. Lehman RA Jr, Kang DG, Lenke LG, Gaume RE, Paik H : The ventral lamina and superior facet rule: a morphometric analysis for an ideal thoracic pedicle screw starting point. Spine J 14 : 137-144, 2014

17. Luther N, lorgulescu JB, Geannette C, Gebhard H, Saleh T, Tsiouris AJ, et al. : Comparison of navigated versus non-navigated pedicle screw placement in 260 patients and 1434 screws: screw accuracy, screw size, and the complexity of surgery. J Spinal Disord Tech 28 : E298-E303, 2015

18. Moussazadeh N, Rubin DG, McLaughlin L, Lis E, Bilsky MH, Laufer I : Short-segment percutaneous pedicle screw fixation with cement augmentation for tumor-induced spinal instability. Spine J 15 : 1609-1617, 2015

19. Nasser R, Drazin D, Nakhla J, Al-Khouja L, Brien E, Baron EM, et al. : Resection of spinal column tumors utilizing image-guided navigation: a multicenter analysis. Neurosurg Focus 41 : E15, 2016

20. Parker SL, McGirt MJ, Farber SH, Amin AG, Rick AM, Suk I, et al. : Accuracy of free-hand pedicle screws in the thoracic and lumbar spine: analysis of 6816 consecutive screws. Neurosurgery 68 : 170-178; discussion 178, 2011

21. Rajasekaran $S$, Kamath $V$, Shetty AP : Intraoperative Iso-C three-dimensional navigation in excision of spinal osteoid osteomas. Spine (Phila Pa 1976) 33 : E25-E29, 2008

22. Shin BJ, James AR, Njoku IU, Härtl R : Pedicle screw navigation: a systematic review and meta-analysis of perforation risk for computernavigated versus freehand insertion. J Neurosurg Spine 17 : 113-122, 2012 